\title{
Impact of oral decontamination on acute graft-versus-host disease and overall survival in children undergoing allogeneic hematopoietic cell transplantation
}

(0) 2020 Polish Society of Hematology and Transfusion Medicine, Insitute of Hematology and Transfusion Medicine. Published by Sciendo. All rights reserved.

\section{Introduction}

Acute graft-versus-host disease (aGVHD) is one of the major causes of morbidity and mortality in children undergoing allogeneic hematopoietic cell transplantation (allo-HCT). Despite advanced methods of its prevention by optimizing donor matching with high-resolution DNA typing of human leukocyte antigens (HLA) and by improved prophylaxis with pharmacological compounds, the incidence of severe aGVHD still occurs in approximately $15-50 \%$ of children following allo-HCT $[1,2]$. aGVHD is considered to develop from donor T-cell activation and recipient tissue inflammation. The inflammatory process can initiate from the whole microorganisms or even parts of bacteria and fungi which may translocate via the damaged mucosal barrier of the host gastrointestinal (GI) tract due to increased permeability after conditioning [3]. Thus, patients undergoing allo-HCT have microbiota disruption, defined as loss of diversity and expansion of pathogenic bacteria [4], which might result in higher mortality [5]. Data on adult patients after alloHCT showed that gut colonization with multidrugresistant (MDR) bacteria was associated with a significantly decreased overall survival (OS) because of an increased treatment-related mortality [6]. The factors that were responsible for this outcome included an increased rate of bacterial infections as well as a higher incidence of aGVHD. Based on the previous reports, within Polish Society of Pediatric Oncology and Hematology, we decided to use oral GI decontamination to suppress the intestinal tract microorganisms [7].
The objective of this study was to analyze whether the use of GI decontamination during the peritransplant period influenced the prevention of aGVHD and had an impact on OS in children undergoing allo-HCT.

\section{Methods}

\section{Design of the study}

All patients who underwent allo-HCT between 2011 and 2014 in pediatric transplant centers belonging to Polish Society of Pediatric Oncology and Hematology were analyzed retrospectively for the impact of oral gut decontamination on aGVHD and OS.

\section{Transplant procedures}

Transplants were conducted according to local procedures and treatment protocols. Toxicities were graded with the use of the toxicity criteria of the Common Terminology Criteria for Adverse Events. aGVHD and chronic GVHD were diagnosed and graded according to standard criteria $[8,9]$.

\section{Colonization}

Most of the patients were routinely screened for $\mathrm{GI}$ colonization of the following MDR bacteria: methicillinresistant Staphylococcus aureus (MRSA), vancomycinresistant enterococcus (VRE), extended spectrum $\beta$-lactamase (ESBL)-producing Enterobacteriaceae, and carbapenemase-producing Enterobacteriaceae. MDR bacteria were determined microbiologically according to the criteria by Magiorakos et al. [10].
Article history:

Received: 03.05.2020 Accepted: 26.05 .2020

Krzysztof Czyżewski', Malgorzata Salamonowicz ${ }^{2}$ Jowita Frączkiewicz ${ }^{2}$, Olga Zajac-Spychała ${ }^{3}$, Jolanta Goździk ${ }^{4}$ Agnieszka Zaucha-Prażmo ${ }^{5}$, Jan Styczyński ${ }^{1, *}$

Department of Pediatric Hematology and Oncology, Collegium Medicum in Bydgosscz Nicolaus Copernicus University in Toruń, Bydgoszcz, Poland 2Department of Pediatric Transplantation, Oncology and Hematology, Medical University, Wroclaw, Poland and Transplantology, University of Medical Sciences Poznań, Poland

Department of Clinical Immunology and Transplantology, University Children's Hospital Jagiellonian University Medical College, Kraków Poland

${ }^{*}$ Corresponding author: Jan Styczyński, Department of Pediatric Hematology and Oncology, Collegium Medicum, Nicolaus Copernicus University Toruń, Skłodowskiej-Curie 9, 85-094 Bydgoszcz, Poland, phone: +48 525854860, fax: +48 525854087, e-mail: jstyczynski@cm.umk.pl 


\section{Prophylaxis of infections}

All patients stayed in a transplant ward with protective environment from the beginning of conditioning until about 1 month after HCT $[11,12]$ and standard of care was applied including prophylaxis of infections and supportive therapy. Most children were nonselectively given antimicrobials for suppression of potentially pathogenic gut bacteria, except those with inability to swallow the drugs. Oral GI decontamination was performed with the use of various drugs including oral ciprofloxacin, metronidazole, colistin, gentamicin, rifaximin, or rarely other compounds. The decontamination was started with the beginning of conditioning and usually finished between neutrophil engraftment and hospital discharge after allo-HCT. For antifungal prophylaxis, up to 2014 fluconazole was usually administered, and later posaconazole or voriconazole was used. Acyclovir was applied for prophylaxis of herpes simplex/varicella-zoster (HSV/ VZV) infections until 1 year after HCT. Preemptive monitoring and treatment strategy were performed for cytomegalovirus (CMV) and Epstein-Barr virus (EBV) reactivation. Prevention of Pneumocystis jiroveci pneumonia (PcP/PjP) was done with cotrimoxazole after neutrophil recovery until the end of immunosuppressive therapy.

\section{Bioethical issues}

All parents provided informed consent for allo-HCT, data analysis, and publication. Collection of rectal swabs was a standard practice in all hospitalized transplant patients according to the local guidelines. This study was approved by the Local Bioethical Committee as a part of a project of analysis of infectious complications in patients undergoing oncological or transplant treatment.

\section{Statistical analysis}

The primary end point of this study was OS after allo-HCT. Deaths from any cause were considered events. Surviving patients were censored at the last follow-up examination or at the date of subsequent alloHCT. Occurrence of aGVHD was a secondary end point. Incidence of aGVHD was calculated using the cumulative incidence method. Survival estimates were calculated using the Kaplan-Meier method and compared by the log-rank test. The impact of the use of oral antibiotics used in gut decontamination on aGVHD was performed with a multivariate logistic regression analysis. All statistical analyses were performed using SPSS version 25.0 (SPSS Inc., Chicago, IL).

\section{Results}

\section{Demographics}

Total number of 459 children (176 girls and 283 boys) at median age 9.3 years (range: $0.1-18$ ) after allo-HCT were included in this study. Children were treated for acute leukemia $(n=343)$, lymphoma $(n=19)$, MDS $(n=23)$, nonmalignant disorders $(n=47)$, and other diseases $(n=26)$, and $74.9 \%$ of patients were in complete remission. Myeloablative conditioning was applied in $73.8 \%$ of HCTs. The source of graft was peripheral blood in $52.9 \%$, bone marrow in $44.7 \%$, and cord blood in $2.4 \%$. Graft was obtained from matched family donor in $25.7 \%$, matched unrelated donor in $54.9 \%$, mismatched unrelated donor in $22.6 \%$, and haploidentical in $4.8 \%$. GVHD prophylaxis was based on cyclosporin A in $92.6 \%$, and rarely on tacrolimus or mycofenolate mofetil.

\section{Decontamination}

Decontamination with antibiotics was applied with 1-3 drugs (including combined or sequential administration) in $78 \%$ of children with oral ciprofloxacin (164 patients; $35.7 \%$ ), metronidazole (86 patients; 18.8\%), colistin (231 patients; 50.3\%), gentamicin (92 patients; $20.0 \%$ ), rifaximin (17 patients; $3.7 \%$ ), or other compounds (15 patients; $3.2 \%$ ).

\section{Outcomes}

The median time to neutrophil and platelet engraftment was 17 (range: 9-60) and 19 (range: 0-516) days, respectively. Mucositis occurred in $90.4 \%$ of patients, aGVHD in $43.4 \%$ including aGVHD II-IV in $55 \%$ of them. GI aGVHD occurred in $16.7 \%$ and cGVHD in $13.4 \%$ of 448 evaluable patients.

A 100-day and 1-year OS were $0.884 \pm 0.015$ and $0.787 \pm 0.019$, respectively. Decontamination had no impact on OS $(p=0.11)$, neither as a strategy nor for any individual drug. Also, decontamination had no impact on incidence of aGVHD $(p=0.3)$, however, it significantly decreased incidence of GI aGVHD ( $p=0.030)$ (Fig. 1). No impact of decontamination on incidence of other organ involvement, including acute skin GVHD, was found.

\section{Impact of antibiotics on GI aGVHD}

In a multivariate logistic regression analysis, any aGVHD increased occurrence of $\mathrm{GI}$ aGVHD $(p<0.001)$, while decontamination had inhibiting effect on GI aGVHD ( $p=0.037)$. In analysis of drugs used in decontamination, the following antibiotics significantly contributed to decreased GI aGVHD: ciprofloxacin $(p<0.05)$, metronidazole $(p<0.05)$, colistin $(p<0.05)$, and gentamicin $(p<0.05)$.

\section{Discussion}

This study was aimed to analyze the impact of gut decontamination on the development of aGVHD and OS in children after allo-HCT. Most of the patients transplanted in pediatric centers received oral decontamination with antibiotics. We have found that decontamination had no impact on OS and aGVHD incidence in this heterogeneous cohort of patients. However, we have shown that gut decontamination reduced the incidence of GI aGVHD. This observation supports gut decontamination before and after allo-HCT to reduce GVHD incidence and severity. Avoiding gut GVHD and mucositis might result in decrease of diarrhea episodes and lead to better tolerance of oral meals, less requirements for total parenteral nutrition, and improved overall quality of life.

It is already well-documented that the gut microbiome exerts immunomodulatory effect after allo-HCT $[5,13]$. With respect to antibiotics, it has been shown previously that decontamination with the use of metronidazole before allo-HCT contributed to a decreased 

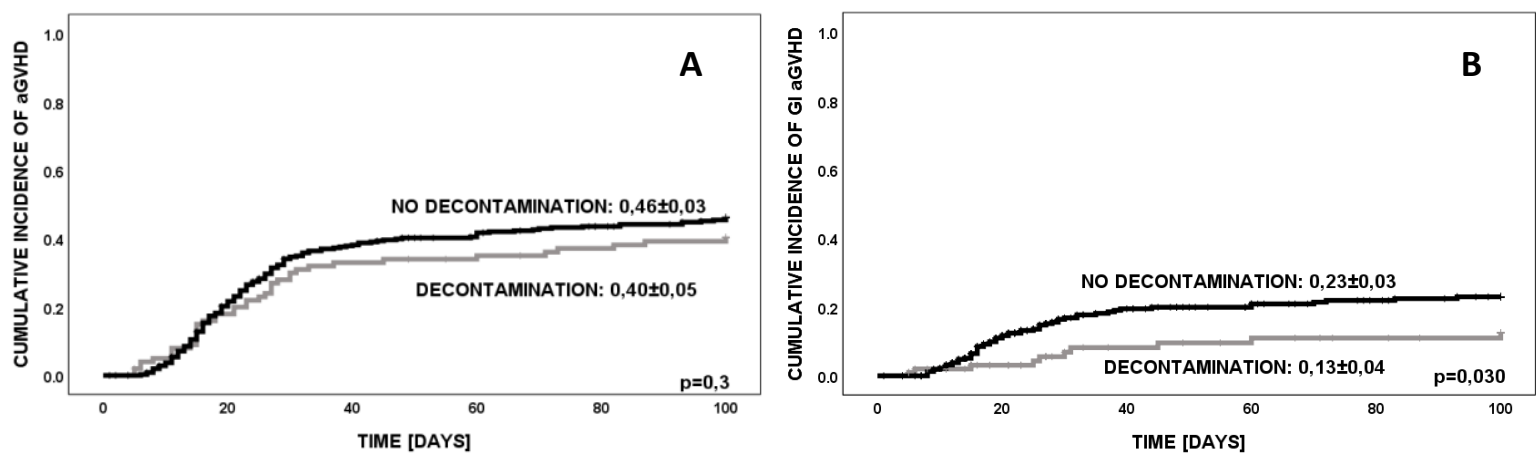

Fig. 1. Impact of decontamination on incidence of acute graft-versus-host disease: (A) overall and (B) gastrointestinal

incidence and severity of aGVHD [14]. However, recent studies linked the use of some antibiotic to the severity and incidence of GVHD: the use of piperacillin - tazobactam or imipenem - cilastatin has led to increased microbiota injury and resulted in increased GVHD severity [15]. Thus, altered gut microbiota, particularly absence or presence of specific bacteria, might be correlated with severity of GVHD and OS. Such findings supported the hypothesis of significant contribution of intestinal bacteria to aGVHD pathophysiology [15].

In our study, we have shown that apart from metronidazole, ciprofloxacin, colistin, and gentamicin might also reduce incidence of acute gut GVHD. Nevertheless, we did not analyze the impact of oral antibiotics on incidence of chronic GVHD, as in the case of chronic GVHD the influence of many immunological factors should also be taken into account. This manuscript presents results of preliminary analyses. Detailed analysis of impact of specific antibiotics is planned by our team.

Gut colonization in patients qualified to treatment with alloHCT probably results from the development of drug-resistance mechanisms during numerous lines of antibiotic treatment used in the treatment of infectious and febrile complications of intensive chemotherapy. This can lead to dissemination of infection including sepsis. Prevention of such complication should be regarded as a standard practice in these severely immunocompromised patients. Although association between intestinal microbiome and transplant outcomes has not been univocally proven, we have shown that oral gut decontamination decreases the incidence of acute gut GVHD.

\section{Acknowledgment}

The authors thank anonymous reviewer for effort to improve the quality of this paper.

\section{Authors' contributions}

JS - study design and administrative support. JS, KC - data analysis and interpretation, statistical analysis, and manuscript writing. All authors - provision of important clinical data, data checkup, and final approval.

\section{Conflict of interest}

All authors declared no conflict of interest related to this study.

\section{Financial support}

None.

\section{Ethics}

The work described in this article has been carried out in accordance with The Code of Ethics of the World Medical Association (Declaration of Helsinki) for experiments involving humans; EU Directive 2010/63/ EU for animal experiments; Uniform requirements for manuscripts submitted to biomedical journals. 


\section{References}

[1] Shi CR, Huang JT, Nambudiri VE. Pediatric cutaneous graft versus host disease: a review. Curr Pediatr Rev 2017;13:100-10.

[2] Styczynski J, Tridello G, Gil L, et al. Prognostic impact of EpsteinBarr virus serostatus in patients with nonmalignant hematological disorders undergoing allogeneic hematopoietic cell transplantation: the study of Infectious Diseases Working Party of the European Society for Blood and Marrow Transplantation. Acta Haematol Pol 2020;51:73-80.

[3] Ferrara JLM, Chaudhry MS. GVHD: biology matters. Blood Adv 2018;2:3411-7.

[4] Golob JL, Pergam SA, Srinivasan S, et al. Stool microbiota at neutrophil recovery is predictive for severe acute graft vs host disease after hematopoietic cell transplantation. Clin Infect Dis 2017;65:1984-91.

[5] Peled JU, Gomes ALC, Devlin SM, et al. Microbiota as predictor of mortality in allogeneic hematopoietic-cell transplantation. $\mathrm{N}$ Engl J Med 2020;382:822-34.

[6] Bilinski J, Robak K, Peric Z, et al. Impact of gut colonization by antibiotic-resistant bacteria on the outcomes of allogeneic hematopoietic stem cell transplantation: a retrospective, singlecenter study. Biol Blood Marrow Transplant 2016;22:1087-93.

[7] Vossen JM, Heidt PJ, van den Berg H, Gerritsen EJ, Hermans J, Dooren LJ. Prevention of infection and graft-versus-host disease by suppression of intestinal microflora in children treated with allogeneic bone marrow transplantation. Eur J Clin Microbiol Infect Dis 1990;9:14-23.

[8] Glucksberg $H$, Storb R, Fefer A, et al. Clinical manifestations of graftversus-host disease in human recipients of marrow from $\mathrm{HL}-\mathrm{A}$ matched sibling donors. Transplantation 1974;18:295-304.
[9] Shulman HM, Sullivan KM, Weiden PL, et al. Chronic graft-versus-host syndrome in man. A long-term clinicopathologic study of 20 Seattle patients. Am J Med 1980;69:204-17.

[10] Magiorakos AP, Srinivasan A, Carey RB, et al. Multidrug-resistant, extensively drug-resistant and pandrug-resistant bacteria: an international expert proposal for interim standard definitions for acquired resistance. Clin Microbiol Infect 2012;18:268-81.

[11] Styczynski J, Tridello G, Donnelly JP, et al. Protective environment for hematopoietic cell transplant (HSCT) recipients: The Infectious Diseases Working Party EBMT analysis of global recommendations on health-care facilities. Bone Marrow Transplant 2018;53:1131-8.

[12] Styczynski J. Infectious complications in children and adults with hematological malignancies. Acta Haematol Pol 2019;50:167-73.

[13] Manzo VE, Bhatt AS. The human microbiome in hematopoiesis and hematologic disorders. Blood 2015;126:311-8.

[14] Beelen DW, Elmaagacli A, Muller KD, Hirche H, Schaefer UW. Influence of intestinal bacterial decontamination using metronidazole and ciprofloxacin or ciprofloxacin alone on the development of acute graft-versus-host disease after marrow transplantation in patients with hematologic malignancies: final results and long-term follow-up of an open-label prospective randomized trial. Blood 1999;93:3267-75.

[15] Shono Y, Docampo MD, Peled JU, et al. Increased GVHD-related mortality with broad-spectrum antibiotic use after allogeneic hematopoietic stem cell transplantation in human patients and mice. Sci Transl Med 2016;8:339ra71. 